\title{
Kernel-Based Image Representation for Brain MRI Discrimination
}

\author{
D. Cárdenas-Peña, A.M. Álvarez-Meza, and Germán Castellanos-Domínguez \\ Signal Processing and Recognition Group \\ Universidad Nacional de Colombia \\ Campus La Nubia, km 7 via al Magdalena, Manizales, Colombia \\ \{dcardenasp, amalvarezme, cgcastellanosd\}@unal . edu.co
}

\begin{abstract}
Discriminating Magnetic Resonance Images (MRI) allows supporting the analysis of physiological and pathological processes, however, finding MRI relationships posses a challenge when analyzing in voxel-based high-dimensional spaces. We introduce a kernel-based representation approach to support MRI discrimination. In this sense, inherent Inter-Slice Kernel relationship is employed to highlight brain structure distributions. Then, a generalized Euclidean metric is estimated by using a kernel-based centered alignment algorithm to code the correlation between MRI dependencies and prior demographic patient information. The proposed approach is tested on MRI data classification by considering patient gender and age categories. Attained results show that proposed methodology improves data interpretability and separability in comparison to state of the art algorithms based on MRI Voxel-wise features. Therefore, introduced kernel-based representation can be useful to support MRI clustering and similarity inference tasks required on template-based image segmentation and atlas construction.
\end{abstract}

\section{Introduction}

The use of Brain Magnetic Resonance Images (MRI) allows analyzing the influence of physiological and pathological processes on structural or functional properties of brain regions [1]. To this end, accurate segmentation of brain structures must be carried out. However, the region labeling is far from being an easy task due to the presence of image artifacts and low inter-structure contrast [4]. To cope with this issue, the atlas-based segmentation is frequently employed, where a given intensity template is non-rigidly registered to a query image, so that the resulting spatial transformation allows the label propagation from the template into the target image coordinate space [9]. In order to significantly improve segmentation accuracy, conventional atlas-based techniques are complemented by machine learning tools. For instance, Authors in [7] propose to register a set of subject atlases to the target image and the most voted label for each voxel is propagated to the final segmentation of the target. Although the use of mixture of classifiers logarithmically rises achieved accuracy, computational burden also dramatically grows with the number of employed atlases. Worse still, atlas quality biases the accomplished segmentation. In fact, those atlases that are similar to the target data tend to overestimate the segmentation accuracy more than the ones that are less similar. Hence, atlas selection must preserve an appropriate similarity for reducing the computational cost while, at the same time, improving the classification accuracy. 
The most common atlas selection approaches leading with training bias are the following: In [10], a set of head models is computed taking into account demographic categories (like age, ethnicity, gender, and skull size), improving source localization by reducing the bias of anatomically unrepresentative atlases. In [2], an introduced mean shift algorithm is employed to perform atlas stratification to determine whether each population is best represented the considered multi-modal distribution. In [1], a ranked atlas selection is performed by computing image similarities among subject images based on measures like sums of squared differences (SSD), cross-correlation, or mutual information. In any case, those estimators do not guarantee convergence due to the involved highly-dimensional spaces.

Here, to improve MRI discrimination, we propose a new kernel-based representation based on the computed inherent Inter-Slice Kernel (ISK) relationship that makes prominent brain structure distributions. Specifically, we compare three different types of ISK-based feature representation to estimate pairwise MRI similarities using generalized Euclidean metrics. We tune all needed metric parameters by means of a centered alignment approach, so that the obtained kernels resemble the most prior demographic information [5[3]. The proposed approach is tested on MRI data discrimination using patient demographic information categories (namely, age and gender patient). As a result, our proposed discriminative representation prove to be useful for MRI clustering tasks, while properly supporting atlas selection approaches.

\section{Materials and Methods}

\subsection{MRI Representation Based on Inter-Slice Similarities}

A 3D Magnetic Resonance Image (MRI) volume comprises a spatially structured set of intensity voxels $\Psi=\left\{x_{\boldsymbol{r}} \in \mathbb{R}: \boldsymbol{r}=(i, j, k)\right\}$, where $x_{\boldsymbol{r}}$ is the magnetic field intensity measured at location $\boldsymbol{r} \in \boldsymbol{R} \subset \mathbb{N}^{3}$. Provided this spatial structure, we describe the MRI volume as an ordered set of $2 \mathrm{D}$ slices along the axis views as:

$$
\Psi=\left\{\boldsymbol{X}_{i}^{v} \in \mathbb{R}^{L_{v^{\prime}} \times L_{v^{\prime \prime}}}: i \in\left\{1, \cdots, L_{v}\right\}\right\}
$$

where $v$ is each one of the considered axes, noted as: axial, a, sagittal $-s$, and coro$n a l-c, i$ indexes the slices, $L_{v}$ corresponds to the volume size in the considered axis and $L_{v^{\prime}}, L_{v^{\prime \prime}}$ are volume sizes in the remaining axes. The arrangement in Eq. (1) provides a useful way to analyze MRIs by medical specialists since to read information on the whole $3 \mathrm{D}$ volume is harder than on a single 2D slice. So, we take advantage of this introduced slice view and propose the use of the Inter-Slice Kernel (ISK) to encode pairwise similarities of the image slice set in the vector, $\boldsymbol{s}^{v} \in \mathbb{R}^{P_{v}}$, with elements described as: $s_{i j}^{v}=\kappa_{\boldsymbol{X}}\left\{\mathrm{d}_{\mathrm{X}}\left(\boldsymbol{X}_{i}^{v}, \boldsymbol{X}_{j}^{v}\right): \forall i<j\right\}$, where $\mathrm{d}_{\mathrm{X}}: \mathbb{R}^{L_{v}^{\prime} \times L_{v}{ }^{\prime \prime}} \times \mathbb{R}^{L_{v}^{\prime} \times L_{v}{ }^{\prime \prime}} \rightarrow \mathbb{R}$ is a used distance operator for implementing the positive definite kernel function $\kappa_{X}\{\cdot\}$, and $P_{v}=L_{v}\left(L_{v}-1\right) / 2$, so $\boldsymbol{s}^{v}$ becomes the ISK representation of the image $\Psi$ along each axis $v$. It is worth noting that the ISK representation becomes much smaller than the original image space, i.e., $L_{v}\left(L_{v}-1\right) / 2 \ll L_{v} L_{v^{\prime}} L_{v^{\prime \prime}}$. 


\subsection{Learning MRI Similarities by Kernel Centered Alignment}

We establish an MRI Similarity Kernel (MSK), $\boldsymbol{K}^{v} \in \mathbb{R}^{N \times N}$ from a set of MRI volumes $\left\{\Psi_{n}: n \in\{1, \ldots, N\}\right\}$, that is the ISK matrix version representing high-dimensional image information along the axes, where $N$ is the number of considered MRIs. Specifically, we perform MRI similarities, for every axis $v$, by computing each pairwise relationship, $k_{n m}^{v} \in \boldsymbol{K}^{v}$, between the ISK-based features as:

$$
k_{n m}^{v}=\kappa_{s}\left\{\mathrm{~d}_{\mathrm{SA}}\left(\boldsymbol{s}_{n}^{v}, \boldsymbol{s}_{m}^{v}\right)\right\}: n, m \in\{1, \ldots, N\}
$$

where $\mathrm{d}_{\mathrm{SA}}: \mathbb{R}^{P_{v}} \times \mathbb{R}^{P_{v}} \rightarrow \mathbb{R}$ is a certain a distance operator implementing the positive definite kernel function $\kappa_{s}\{\cdot\}$. In order to reveal the main ISK relationships for learning MRI similarities, we rely on the Mahalanobis distance defined in $P_{v}$-dimensional space with inverse covariance matrix $\boldsymbol{A}^{v} \boldsymbol{A}^{v \top}$ as:

$$
\mathrm{d}_{\mathrm{SA}}{ }^{2}\left(\boldsymbol{s}_{n}^{v}, \boldsymbol{s}_{m}^{v}\right)=\left(\boldsymbol{s}_{n}^{v}-\boldsymbol{s}_{m}^{v}\right) \boldsymbol{A}^{v} \boldsymbol{A}^{v \top}\left(\boldsymbol{s}_{n}^{v}-\boldsymbol{s}_{m}^{v}\right)^{\top} .
$$

where matrix $\boldsymbol{A}^{v} \in \mathbb{R}^{P_{v} \times D_{v}}$ holds the linear projection $\boldsymbol{v}_{n}^{v}=\boldsymbol{s}_{n}^{v} \boldsymbol{A}^{v}$, with $\boldsymbol{v}_{n}^{v} \in \mathbb{R}^{D_{v}}$ and $D_{v} \leq P_{v}$. Moreover, we propose to learn the matrix $\boldsymbol{A}^{v}$ based on the already estimated ISK-based feature similarities and by adding prior demographic patient information enclosed in the matrix $\boldsymbol{B} \in \mathbb{R}^{N \times N}$. Thus, we measure the dependence between both matrices $\boldsymbol{K}^{v}$ and $\boldsymbol{B}$ through the following kernel target centered alignment function [3[5]:

$$
\rho\left(\boldsymbol{K}^{v}, \boldsymbol{B}\right)=\frac{\left\langle\boldsymbol{H} \boldsymbol{K}^{v} \boldsymbol{H}, \boldsymbol{H} \boldsymbol{B} \boldsymbol{H}\right\rangle_{F}}{\left\|\boldsymbol{H} \boldsymbol{K}^{v} \boldsymbol{H}\right\|_{F}\|\boldsymbol{H} \boldsymbol{B} \boldsymbol{H}\|_{F}}, \rho \in[0,1]
$$

where $\boldsymbol{H}=\boldsymbol{I}-N^{-1} \mathbf{1 1}^{\top}$, with $\boldsymbol{H} \in \mathbb{R}^{N \times N}$, is a centering matrix, $\mathbf{1} \in \mathbb{R}^{N}$ is an all-ones vector, and notations $\langle\cdot, \cdot\rangle_{F}$ and $\|\cdot, \cdot\|_{F}$ stand for the Frobenius inner product and norm, respectively. Generally, the centered version of the alignment coefficient in Eq. (4) gets better correlation estimates than its uncentered version [5]3].

Therefore, we propose to learn MRI similarities from ISK-based features taking advantage of the Kernel Center Alignment (KCA) cost function described in Eq. (4). In this sense, prior patient information, e.g, demographic data as age and gender, can be employed to reveal MRIs dependencies by learning the matrix $\boldsymbol{A}^{v}$ that parameterizes a Mahalanobis distance between pairwise images (see Eq. (3)). Thereby, given a demographic-based similarity matrix $\boldsymbol{B}$, a KCA-based function can be formulated to compute the projection matrix $\boldsymbol{A}^{v}$ in Eq. (2) as:

$$
\boldsymbol{A}^{v *}=\underset{\boldsymbol{A}^{v}}{\operatorname{argmax}} \rho\left(\boldsymbol{K}_{\boldsymbol{A}^{v}}^{v}, \boldsymbol{B}\right),
$$

where $\boldsymbol{K}_{\boldsymbol{A}^{v}}^{v}$ is the resulting MSK matrix for a provided $\boldsymbol{A}^{v}$ projection as given in Eq. (2). Consequently, we term each $\boldsymbol{K}_{\boldsymbol{A}^{v *}}^{v}$ as a Learned MRI Similarity Matrix (LMSK).

\section{Experiments and Results}

\subsection{Demographic MRI Database and Preprocessing}

The IXI dataset is a brain imaging study holding MR images from 575 normal subjects aging between 20 and 80 years. Subjects were provided with T1, T2, PD, DTI, and 
angiogram volumes. All image sequences were acquired with three different scanners (Philips 1.5T, Philips 3T, and GE 3T), to be further anonymised and converted to NIFTI format. Additionally, basic demographic information for each subject is included (age, gender, ethnicity, among others). The whole dataset is publicly available online 1 . Since the current paper goal concerns the atlas construction, the T1 sequences were only taken into account which were acquired with a GE 3 T scanner fro $N=314$ subjects (139 male and 175 female). Each T1 sequence comprises $256 \times 256 \times 150$-sized volumes with a voxel size of $0.9375 \times 0.9375 \times 1.2 \mathrm{~mm}$.

We perform two preprocessing steps over all image data: Firstly, each image is registered to the MNI305 template by an affine transform to reference the whole dataset to the Talairach space. After registering, each volume is re-sampled to $197 \times 233 \times 189$ size. Secondly, an intensity normalization is performed by scaling each voxel value, so that the mean intensity of the white matter is equal to 110 [6]. We carry out both preprocessing steps with the Free surfer image analysis suite freely available online 2 .

\subsection{MRI Discrimination Using LMSK}

We explore three similarity-based image representation techniques to estimate patient patterns from MRI. The first one is the baseline Sum of Squared Differences (SSD), where each image voxel is used as feature [1]. The second one uses the proposed MSK representation in its plain form, i.e., we assume $\boldsymbol{A}^{v}=\boldsymbol{I}$ in Eqs. (2) and (3). The last one uses the more elaborate LMSK version by learning an MRI Mahalanobis distance as in Eq. (4). With regard to the needed kernel functions, because of its universal approximating capability [8], we choose the well-known Gaussian kernel noted as follows:

$$
g\left\{\mathrm{~d}_{\mathrm{z}}\left(z, z^{\prime}\right) ; \sigma\right\} \triangleq \exp \left(-\mathrm{d}_{\mathrm{z}}\left(z, z^{\prime}\right)^{2} /\left(2 \sigma^{2}\right)\right)
$$

where $\sigma \in \mathbb{R}^{+}$is the kernel bandwidth; $z, z^{\prime} \in Z$ is a sample pair in a given feature space $z$, and $\mathrm{d}_{\mathrm{z}}: Z \times z \rightarrow \mathbb{R}$ is a distance operator in $z$. Thus, for the SSD approach, we calculate each element $y_{n m}$ of the MRI similarity matrix $\boldsymbol{Y} \in \mathbb{R}^{N \times N}$ using the Euclidean metric between $n$-th and $m$-th images as:

$$
y_{n m}=g\left(\left\|\operatorname{vec}\left(\Psi_{n}\right)-\operatorname{vec}\left(\Psi_{m}\right)\right\|_{2} ; \sigma_{V}\right),
$$

where notation $\operatorname{vec}(\cdot)$ stands for column-based concatenation of the argument matrix and $\|\cdot\|_{2}$ stands for the 2-norm. In turn, we calculate each ISK-based feature vector $\boldsymbol{s}^{v}$ from MRI using the Frobenious norm:

$$
s_{i j}^{v}=g\left(\left\|\boldsymbol{X}_{i}^{v}-\boldsymbol{X}_{j}^{v}\right\|_{F} ; \sigma_{s_{v}}\right) .
$$

Afterwards, we calculate each $\boldsymbol{K}^{\nu}$ matrix encoding pairwise MRI relationship as in Eq. (2), yielding:

$$
k_{n m}^{v}=g\left(\mathrm{~d}_{\mathrm{SA}}\left(\boldsymbol{s}_{n}^{v}, \boldsymbol{s}_{m}^{v}\right) ; \sigma_{S A_{v}}\right) .
$$

\footnotetext{
${ }^{1}$ http://www . brain-development .org/

${ }^{2}$ http://surfer.nmr.mgh.harvard.edu/
} 
We optimize the KCA-based cost function in Eq. (5) to learn $\boldsymbol{A}^{v}$ by a gradient descent solver, where the initial feasible solution is calculated by the Principal Component Analysis algorithm. In addition, the elements of the label kernel $\boldsymbol{B}$ are set as: $b_{n m}=\delta\left(c_{n}-c_{m}\right)$, being $\delta$ the delta function and where $c_{n} \in\{1,2, \ldots, C\}$ is the label of $\Psi_{n}$. Namely, four classes are considered from the IXI dataset: adult male, senior male, adult female, and senior female, with adult label corresponding people aging between 20 and 50 years, and senior label - aging more than 50 years old. It is worth noting that every kernel bandwidth in Eqs. (6) to (8) must be properly tuned. Since the variability of the Gaussian kernel $g(\cdot ; \sigma)$ tends to zero whenever the kernel bandwidth tends to either zero or infinity to get an appropriate $\sigma$ value spanning widely all similarity values, we propose to adjust the Gaussian kernel bandwidth employing the following criterion (Notation $\operatorname{var}(\cdot)$ stands for the variance operator):

$$
\sigma^{*}=\arg \max _{\sigma}\{\operatorname{var}(g(\cdot ; \sigma)\}
$$

Figs. 1(a) to 1(c) show a concrete MRI example illustrating all three views. As seen in Figs. 1(d) to 1(f) displaying their corresponding estimated ISK representations, the red corner patches keep the MRI edges with no content, i.e., the background. Moreover, as the Sagittal ISK (see Fig. 1(f)) exhibits symmetry respect to the anti-diagonal, it is clear that such representation is able to keep the head sagittal symmetry. Therefore, due to the kernel shape varies accordingly to the brain structure distribution, we infer that proposed ISK suitably characterizes head shapes. Although the latent phenomenon is the same for all ISK, each of them are providing a different view of the patient brain structures. Concerned the accomplished MRI similarities, the upper row of Fig. 2 shows the attained SSD and MSK-based kernels by fixing the bandwidth as in Eq. (9), while the lower row shows the LMSK kernels adjusted to the label kernel in Fig. 2(e). Detailed visual inspection of the estimated MRI similarities allows noting that the considered demographic categories can not be not easily distinguished from the baseline representation (see Fig. 2(a)). Instead, the computed MSK kernels discover a blocked structure according to the demographic information. Therefore, despite the unsupervised nature of the MSK kernel, the proposed ISK feature extraction is useful to perform an MRI clustering into demographic categories. Moreover, the LMSK kernels that are computed in a supervised scheme have a structure similar to the label kernel, as expected. Thus, LMSK is able to find a linear projection that makes more prominent the main correlations between MRI similarities and demographic categories.

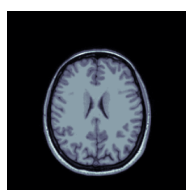

(a) Axial

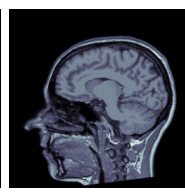

(b) Sagittal

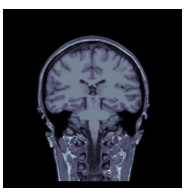

(c) Coronal

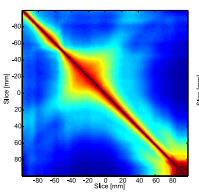

(d) Axial

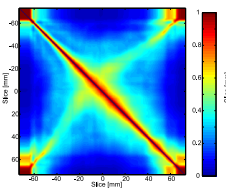

(e) Coronal

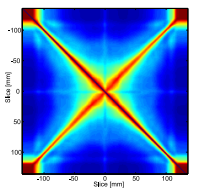

(f) Sagittal

Fig. 1. IXI database-Subject 002 and their estimated ISK representation per view 


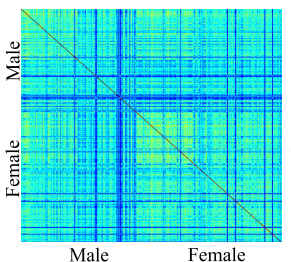

(a) SSD

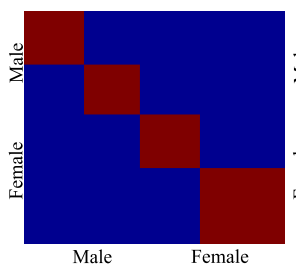

(e) Label kernel

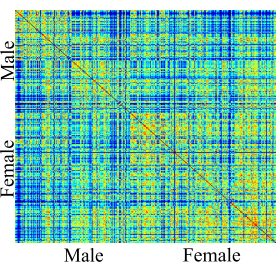

(b) $\mathrm{MSK}_{\mathrm{a}}$

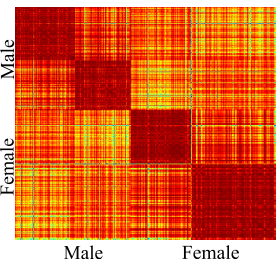

(f) $\mathrm{LMSK}_{\mathrm{a}}$

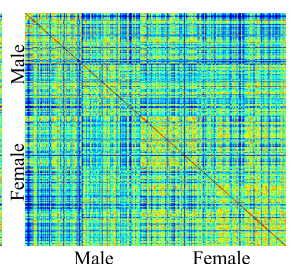

(c) $\mathrm{MSK}_{\mathrm{c}}$

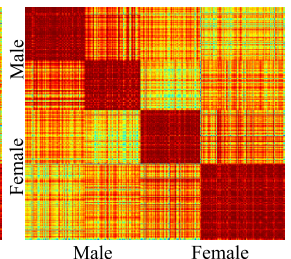

(g) $\mathrm{LMSK}_{\mathrm{c}}$

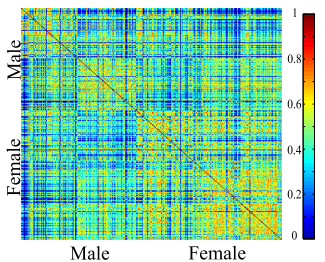

(d) $\mathrm{MSK}_{\mathrm{s}}$

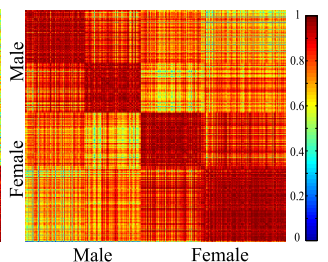

(h) $\mathrm{LMSK}_{\mathrm{s}}$

Fig. 2. Estimated MRI similarities

For the sake of visual comparison, the 3D Kernel Principal Component Analysis (KPCA) projection is applied on SSD and $\mathrm{LMSK}_{\mathrm{c}}$ MRI similarities. As seen in Fig. 3, our proposal provides better class separability than the SSD baseline. Moreover, the $\mathrm{LMSK}_{\mathrm{c}}$ projection axes provides information about each considered category. Due to the observed better separation between male and female classes in the second decomposition than in the others, this axis is related to the gender category. Similarly, the third component allows to separate between different ages, exhibiting a linear increasing pattern from adult to senior.

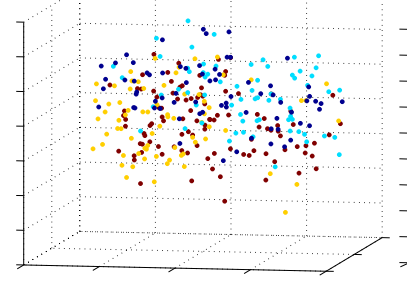

(a) SSD

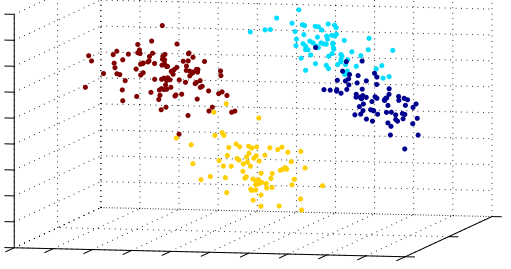

(b) $\mathrm{LMSK}_{\mathrm{c}}$

Fig. 3. 3D projections. $\bullet$ adult male $\bullet$ senior male $\bullet$ adult female $\bullet$ senior female

To validate these important findings, a 1-nearest neighbor classifier is trained using each kernel-based induced distance from MRI similarities (see Figs. 1(d) to 1(f)), where we provide the 10-fold cross-validation strategy for validation purposes. As seen in Table 1 , the proposed MSK is able to enhance SSD performance when classifying Gender categories. Although Gender SSD accuracy can be considered as acceptable, it should 
be noted that its obtained representation resides on a higher dimensional space representation that the one using ISK. Regarding to Gender-Age categories MSK (Coronal view) outperforms considered unsupervised MRI dependencies whereas SSD achieves unstable classification results. Also, as seen in Table 1, the proposed LMSK-based representations allow to reveal suitable linear projection that facilitates MRIs discrimination by taking advantage of patient demographic information. Thus, similar brain structures are coded by KCA from MRIs and demographic kernel correlations. Finally, Tables 2 to 3 presents the confusion matrices for SSD and $\mathrm{LMSK}_{\mathrm{c}}$ based approaches. SSD approach it is able to obtain an acceptable gender discrimination, particularly, for female label, however, it is not able to distinguish age categories. So, complex brain structures, e.g., those related with patient age variations, can not be encode by SSD.

Table 1. Gender and Gender-Age accuracy results[\%]

\begin{tabular}{|c|c|c|c|c|c|c|c|}
\hline Category & SSD & MSK $_{\mathrm{a}}$ & MSK $_{\mathrm{c}}$ & MSK $_{\mathrm{s}}$ & LMSK $_{\mathrm{a}}$ & LMSK $_{\mathrm{c}}$ & $\mathrm{LMSK}_{\mathrm{s}}$ \\
\hline $\mathrm{G}$ & $77.3 \pm 07.0$ & $77.0 \pm 04.5$ & $80.8 \pm 07.3$ & $\mathbf{8 2 . 8} \pm \mathbf{0 6 . 0}$ & $99.3 \pm 01.3$ & $\mathbf{1 0 0} \pm \mathbf{0}$ & $\mathbf{1 0 0} \pm \mathbf{0}$ \\
GA & $55.2 \pm 12.2$ & $52.2 \pm 04.3$ & $55.1 \pm 04.8$ & $\mathbf{5 9 . 9} \pm \mathbf{0 5 . 5}$ & $93.9 \pm 02.8$ & $\mathbf{9 7 . 4} \pm \mathbf{0 2 . 0}$ & $88.8 \pm 06.9$ \\
\hline
\end{tabular}

Table 2. SSD confusion matrix [\%]

\begin{tabular}{|c|c|c|c|c|}
\hline Category & $\begin{array}{c}\text { adult } \\
\text { male }\end{array}$ & $\begin{array}{c}\text { senior } \\
\text { male }\end{array}$ & $\begin{array}{c}\text { adult } \\
\text { female }\end{array}$ & $\begin{array}{c}\text { senior } \\
\text { female }\end{array}$ \\
\hline $\begin{array}{c}\text { adult } \\
\text { male }\end{array}$ & 55.4 & 43.6 & 08.4 & 05.9 \\
\hline $\begin{array}{c}\text { senior } \\
\text { male }\end{array}$ & 11.0 & 30.2 & 01.4 & 08.8 \\
\hline $\begin{array}{c}\text { adult } \\
\text { female }\end{array}$ & 21.1 & 10.2 & 72.1 & 26.0 \\
\hline $\begin{array}{c}\text { senior } \\
\text { female }\end{array}$ & 12.5 & 15.9 & 18.1 & 59.3 \\
\hline
\end{tabular}

Table 3. $\mathrm{LMSK}_{\mathrm{c}}$ confusion matrix [\%]

\begin{tabular}{|c|c|c|c|c|}
\hline Category & $\begin{array}{c}\text { adult } \\
\text { male }\end{array}$ & $\begin{array}{c}\text { senior } \\
\text { male }\end{array}$ & $\begin{array}{c}\text { adult } \\
\text { female }\end{array}$ & $\begin{array}{c}\text { senior } \\
\text { female }\end{array}$ \\
\hline $\begin{array}{c}\text { adult } \\
\text { male }\end{array}$ & 98.6 & 01.4 & 0 & 0 \\
\hline $\begin{array}{c}\text { senior } \\
\text { male }\end{array}$ & 01.4 & 98.6 & 0 & 0 \\
\hline $\begin{array}{c}\text { adult } \\
\text { female }\end{array}$ & 0 & 0 & 94.7 & 01.9 \\
\hline $\begin{array}{c}\text { senior } \\
\text { female }\end{array}$ & 0 & 0 & 05.4 & 98.1 \\
\hline
\end{tabular}

\section{Conclusions}

A kernel-based image representation is introduced to support MRI discrimination in segmentation of brain structures. Our proposal encodes inter-slice variations related to the brain structure distribution. Thus, head patterns can be extracted along each 3D axis view, namely, Axial, Coronal, and Sagittal. Furthermore, a kernel-based centered alignment strategy is carried out to explore the correlation between MRI-based similarities and patient demographic information. Therefore, a parameterized Euclidean distance is learned for enhancing both data interpretability and separability to facilitate MRI discrimination. Taking into account the attained results over a well-know MRI dataset, the proposed kernel-based representation methodology proves to find the natural inherent distributions of MRI, namely, age and gender categories. In addition, the proposed methodology improves data separability in comparison to state of the art algorithms based on Voxel-wise representation. So, our proposal is suitable to support 
MRI classification and similarity measurement tasks required on template-based image segmentation. As future work, authors plan to test our approach as a template subset selector on MRI segmentation tasks, so that the structure classification results can be improved by taking advantage of patient demographic data. Moreover, other information theory-based distance should be of benefit for dealing with MRI data.

Acknowledgments. This work was supported by Programa Nacional de Formación de Investigadores “Generación del Bicentenario”, 2011/2012 and project Evaluación asistida de potenciales evocados cognitivos como marcador del trastorno por déficit de atención e hiperactividad (TDAH) - Convocatoria 569 all funded by COLCIENCIAS.

\section{References}

1. Aljabar, P., Heckemann, R.A., Hammers, A., Hajnal, J.V., Rueckert, D.: Multi-atlas based segmentation of brain images: atlas selection and its effect on accuracy. NeuroImage 46(3), 726-738 (2009)

2. Blezek, D.J., Miller, J.V.: Atlas stratification. Medical Image Analysis 11(5), 443-457 (2007)

3. Brockmeier, A., Choi, J., Kriminger, E., Francis, J., Principe, J.: Neural decoding with kernelbased metric learning. Neural Computation 26 (2014)

4. Cardenas-Pena, D., Martinez-Vargas, J.D., Castellanos-Dominguez, G.: Local binary fitting energy solution by graph cuts for MRI segmentation.. EMBC 2013(2), 5131-5134 (2013)

5. Cortes, C., Mohri, M., Rostamizadeh, A.: Algorithms for learning kernels based on centered alignment. The Journal of Machine Learning Research 13, 795-828 (2012)

6. Fischl, B., Salat, D.H., Busa, E., Albert, M., Dieterich, M., Haselgrove, C., van der Kouwe, A., Killiany, R., Kennedy, D., Klaveness, S., Montillo, A., Makris, N., Rosen, B., Dale, A.M.: Whole brain segmentation: automated labeling of neuroanatomical structures in the human brain.. Neuron 33(3), 341-355 (2002)

7. Heckemann, R.A., Hajnal, J.V., Aljabar, P., Rueckert, D., Hammers, A.: Automatic anatomical brain MRI segmentation combining label propagation and decision fusion. NeuroImage 33(1), 115-126 (2006)

8. Liu, W., Principe, J.C., Haykin, S.: Kernel Adaptive Filtering: A Comprehensive Introduction, vol. 57. John Wiley \& Sons (2011)

9. Lötjönen, J.M., Wolz, R., Koikkalainen, J.R., Thurfjell, L., Waldemar, G., Soininen, H., Rueckert, D.: Fast and robust multi-atlas segmentation of brain magnetic resonance images. NeuroImage 49(3), 2352-2365 (2010)

10. Valdés-Hernández, P.A., von Ellenrieder, N., Ojeda-Gonzalez, A., Kochen, S., AlemánGómez, Y., Muravchik, C., Valdés-Sosa, P.A.: Approximate average head models for EEG source imaging. Journal of Neuroscience Methods 185(1), 125-132 (2009) 